\title{
Protective Effect of Tong-Qiao-Huo-Xue Decoction on Inflammatory Injury Caused by Intestinal Microbial Disorders in Stroke Rats
}

\author{
Feng Zhang, ${ }^{a, b, c}$ Mengting Zhai, ${ }^{a}$ Qian Wu, ${ }^{a, b, c}$ Xiaoyi Jia, ${ }^{a, b, c}$ Yan Wang, ${ }^{*, a, b, c}$ and \\ Ning Wang ${ }^{*, a, b, c}$ \\ ${ }^{a}$ Key Laboratory of Chinese Medicinal Formula of Anhui Province, Anhui University of Chinese Medicine; Hefei \\ 230012, China: ${ }^{b}$ Institute for Pharmacodynamics and Safety Evaluation of Chinese Medicine, Anhui Academy of \\ Chinese Medicine; Hefei 230012, China: and ${ }^{c}$ Key Laboratory of Xin'an Medicine, Ministry of Education; Hefei \\ 230012, China.
}

Received September 30, 2019; accepted February 18, 2020; advance publication released online March 3, 2020

Tong-Qiao-Huo-Xue Decoction (TQHXD) is a classic traditional Chinese medicine prescription for treating cerebral ischemia. The purpose of this study was to investigate the effect of TQHXD on intervening inflammatory response of ischemic stroke by regulating intestinal flora and repairing the intestinal barrier. A rat model of cerebral ischemia was established using middle cerebral artery occlusion (MCAO) and behavioral scores were performed. Additionally, the high throughput 16S ribosomal DNA (rDNA) sequence of intestinal bacteria in fecal samples of rat was also carried out. Our results showed that TQHXD could change the main components of intestinal flora in stroke rats, and reduced the excessive increase of Bacteroidetes, and also regulated the abnormal changes of abundance of some flora as well. In addition, the intestinal epithelial barrier was damaged after stroke, allowing bacterial metabolites to enter the blood, while TQHXD had an improved effect on this phenomenon. Meanwhile, pathological changes in the brain tissue and infarct volume were also alleviated by TQHXD. Due to the disorder of the intestinal flora and the destruction of the barrier, the peripheral immune imbalance caused an inflammatory reaction. TQHXD improved the imbalance of $T$ cells, and inhibited the inflammatory response. Finally, the therapeutic transplantation of fecal microbiota also improved the outcome of stroke in rats. Our presented results suggest that TQHXD may improve the gut microbiota disorder and its induced inflammatory response after stroke, which could be a new target and mechanism for the treatment of stroke.

Key words stroke; gut microbiota; inflammatory response; $\mathrm{T}$ cell

\section{INTRODUCTION}

Stroke is an acute cerebrovascular disease that is extremely harmful to human health due to its high disability and mortality rate. ${ }^{1,2}$ Among them, ischemic stroke accounts for more than $80 \%$ of the overall incidence of stroke. ${ }^{3)}$ Related studies have shown that post-stroke inflammatory response is a longlasting pathological process of ischemic brain injury, which is an important cause of secondary brain injury. ${ }^{4)}$ This process is closely related to intestinal microbial imbalance and intestinal barrier destruction after stroke. ${ }^{5-8)}$

Previous reports have shown $\mathrm{T}$ cells play a key role in secondary neuroinflammation following stroke. ${ }^{9-11)} \mathrm{T}$ cells and lymphocytes have also been identified as the main substances in the regulation of inflammatory response after brain injury, especially $\mathrm{T}$ cells play a major role, and regulatory $\mathrm{T}$ cells (Tregs) can inhibit neuroinflammatory damage. ${ }^{11-14)}$ In addition to triggering the activation of local $\mathrm{T}$ cells after stroke, it can also be observed that peripheral immune organ $\mathrm{T}$ cells are also activated. ${ }^{15-17)}$ Related studies have shown that gut microbiota plays an important role in maintaining the homeostasis of T cells. ${ }^{18-20)}$ Recently, "intestine-brain axis" theory of the functional relationship between the intestine and the brain has become a new focus. ${ }^{21-24)}$ Benakis et al. ${ }^{25)}$ found that intestinal microbiota disturbance caused by ischemic stroke caused changes in $\mathrm{T}$ cell expression, and the induction of antibiotic therapy affected the inflammatory response and outcome of the stroke model. Therefore, regulating the imbalance of in- testinal micro-ecology after ischemic stroke is of great significance for inhibiting inflammation and reducing the incidence of disabling and lethality.

Tong-Qiao-Huo-Xue Decoction (TQHXD) is a classical prescription in traditional Chinese medicines, consisted of 7 Chinese herbs (Table 1). TQHXD can promote blood circulation and remove blood stasis. Our research group had established HPLC fingerprints of TQHXD for the quality control, and analyzed the chemical constituents in rat cerebrospinal fluid by GC and HPLC after TQHXD administration. ${ }^{26,27)}$ Previous studies by our team showed that TQHXD has a protective effect on the injury to cerebral ischemia reperfusion rats and the injury to PC12 cells. ${ }^{27,28)}$ At the same time, inflammatory cell infiltration was found in the ischemic hemisphere. In-

Table 1. Drug Composition of TQHXD

\begin{tabular}{llc}
\hline \hline \multicolumn{1}{c}{ Chinese medicine } & \multicolumn{1}{c}{ Family } & Weight (g) \\
\hline 1 She Xiang (Moschus berezovskii & Cervidae & 0.15 \\
FLEROV) & Asteraceae & 9 \\
2 Hong Hua (Carthamus tinctorius L.) & Apiaceae & 3 \\
3 Chuan Xiong (Ligusticum chuanxiong & & \\
HORT) & Ranunculaceae & 9 \\
4 Chi Shao (Paeonia veitchii Lynch) & Rosaceae & 9 \\
5 Tao Ren (Prunus persica (L.) & Rhamnaceae & 5 \\
BATSCH) & Liliaceae & 3 \\
6 Da Zao (Ziziphus jujube Mill) & & \\
7 Cong (Scallion) & &
\end{tabular}


flammation after stroke is closely linked to gut microbes, but whether TQHXD could exert its protective effect on inflammatory injury after ischemic stroke by regulating intestinal microecology is unclear. Therefore, this experiment aims to explore effects of TQHXD to protect ischemic stroke by regulating the gut microbiota, and also provide new ideas of prevention and treatment of ischemic stroke.

\section{MATERIALS AND METHODS}

Reagents The constitutions of TQHXD were purchased from He Yi Tang Traditional Chinese Medicines Group Corporation (Hefei, China). All the medicinal materials were identified as authentic by Prof. Shoujin Liu (Chinese herbal medicine specimen room of Anhui University of Chinese Medicine). Weighed 10 times the weight of the TQHXD original medicinal material, and soaked in 10 times $75 \%$ ethanol for $2 \mathrm{~h}$, and refluxed for $2 \mathrm{~h}$, then filtered and added 8 times of $75 \%$ ethanol with refluxing for $1.5 \mathrm{~h}$, and combined the filtrate. Then $1.5 \mathrm{~g}$ artificial musk was mixed into the extract, and the solution was adjusted to a concentration of $2 \mathrm{~g} / \mathrm{mL} .^{27)}$

Triphenyltetrazolium chloride (TTC) and paraformaldehyde was purchased from Sigma (St. Louis, MO, U.S.A.). Anti-ZO-1, Anti-Claudin-2, $\beta$-actin antibodies were purchased from Abcam (Cambridge, MA, U.S.A.). Anti-Rat CD3, AntiRat CD4, Anti-Rat CD25, Anti-Rat $\gamma \delta$ T-Cell Receptor (TCR) purchased from BD Pharmingen (San Diego, CA, U.S.A.). Anti-Rat Foxp3 were purchased from eBioscience (San Diego, CA, U.S.A.). Ficoll-Paque PREMIUM were purchased from GE Life (U.K.). Recombinant Anti-Foxp3 antibody, Anti-TCR gamma+TCR delta antibody [V65] receptor were purchased from Abcam. Diamine oxidase (DAO), D-lactic and lipopolysaccharides (LPS) Elisa kits purchased from Meimian (Yan Cheng, China). All other chemicals were commercial products of analytical grade.

Animals Male Sprague-Dawley (SD) rats weighing 240-260g were purchased from the Animal Experimental Center of Nantong University, certificate number: (SCXK: Jiangsu 2014-0001). All animal experiments were approved by the Animal Ethics Committee of Anhui University of Chinese Medicine.

Cerebral Ischemia-Reperfusion (I/R) Model Animal model of stroke was established according to Longa's methods. ${ }^{29)}$ Briefly, rats were anesthetized with isoflurane $(3-4 \%$ for anesthesia induction; $1-2 \%$ for anesthesia maintenance). The left common carotid artery (CCA), external carotid artery (ECA) and internal carotid artery (ICA) of the rat were exposed and separated. The CCA and ECA were ligated and the ICA was clamped with an arterial clip. Then cut a small opening from the distal end of the CCA and insert the paraffin-coated $0.285 \mathrm{~mm}$ nylon thread from the CCA into the ICA until the middle cerebral artery (MCA). The procedure for the sham-operated rats was the same as that of the model rats, but did not insert the line into the MCA of the rats. At the end of the surgery, the rats were placed in an incubator at $37 \pm 0.5^{\circ} \mathrm{C}$ until they recovered consciousness. Neurobehavioral scores were performed after $24 \mathrm{~h}$.

Drug Administration To explore the effects of TQHXD on intestinal dysfunction and inflammatory response induced by stroke, rats were randomly divided into 3 groups: sham group, model group, TQHXD group $(6 \mathrm{~g} / \mathrm{kg})$. The model group and the TQHXD group were further divided into two subgroups of $7 \mathrm{~d}$ and $14 \mathrm{~d}$. Twenty-four hours after cerebral ischemia-reperfusion in rats, the TQHXD $7 \mathrm{~d}$ group (tq) and the $14 \mathrm{~d}$ group (TQ) were intragastrically administered at $1 \mathrm{~mL} / 100 \mathrm{~g}$ once daily. At the same time, the model $7 \mathrm{~d}$ group (is) and the $14 \mathrm{~d}$ group (IS) as well as the sham group were given physiological saline. Sampling was performed at two time points, $7 \mathrm{~d}$ and $14 \mathrm{~d}$.

To further verify the role of the gut microbiota throughout the experiment, we first randomly divided the rats into three groups: Sham group, Sham+TQHXD group, and Model+TQHXD group. The Sham+TQHXD group and the Model+TQHXD group were given TQHXD once a day at $1 \mathrm{~mL} / 100 \mathrm{~g}$ for $14 \mathrm{~d}$. After $7 \mathrm{~d}$ of dosing, fecal microbiota transfer (FMT) was performed. Divided into Model group, Sham (FMT) group, Sham+TQHXD (FMT) group, Model+TQHXD (FMT) group. The Sham (FMT) group, the Sham+TQHXD (FMT) group, and the Model+TQHXD (FMT) group were given fresh fecal supernatant once a day for 7 consecutive days. The Model group was given an equal amount of sterile water.

Neurological Score Rats' behavioral scores were determined using the Longa 5 grade 4-point scale to measure neurological deficit scores. ${ }^{11)}$ The standard was as follows: no neurological deficit symptoms $=0$, cannot fully extend the contralateral front paw $=1$, circling to right $=2$, falling to right and difficulties in consciousness $=3$, cannot walk spontaneously and consciousness in coma $=4$.

Hematoxylin-Eosin (H\&E) Staining Two rats per group were anesthetized, and perfused by saline through the heart, then with $4 \%$ paraformaldehyde. The small intestine and brain were removed and placed in paraformaldehyde at $4^{\circ} \mathrm{C}$. Paraffin sections were prepared, subjected to conventional dewaxing, and coronal sections ( $5 \mu \mathrm{m}$ thick) were taken after H\&E staining, and observed under a microscope.

Transmission Electron Microscopy (TEM) The rats were treated as H\&E staining assay. The small intestine tissue was cut into 3 pieces of about $1 \mathrm{~mm}$ and fixed in $2.5 \%$ glutaraldehyde at $4^{\circ} \mathrm{C}$. Then the tissues were placed in $2 \%$ osmium for $1.5 \mathrm{~h}$, dehydrated with gradient alcohol, embedded in epoxy resin, ultrathin sliced, after double staining of uranyl acetate and lead citrate, observation by Transmission Electron Microscopy (TEM-1230, JPN).

Enzyme-Linked Immunosorbent Assay (ELISA) The first step was to detect the concentration of DAO and D-lactic acid in plasma. Eight rats per group were anesthetized. Abdominal aorta blood sampling into heparin anticoagulation tube, centrifuged at $3000 \mathrm{rpm}$ for $10 \mathrm{~min}$ and the supernatant was taken. According to the operation instructions in ELISA kit, the detection methods are as follows: dilute the standard and sample, then add samples according to the group, and warm bath at $37^{\circ} \mathrm{C}$ for $30 \mathrm{~min}$, and wash and add enzyme labeling reagent, and warm bath and wash again, then add developer $\mathrm{A}$ and $\mathrm{B}$ avoiding light for $10 \mathrm{~min}$ at $37^{\circ} \mathrm{C}$. Finally add stop solution, detect at $450 \mathrm{~nm}$ by enzyme-labeled instrument. The concentration of LPS in the plasma was measured except that the endotoxin-specific test tube for heparinized treatment was used, and the other operations were the same as above. In addition, ELISA was used to detect the expression of interleukin (IL)-10 and IL-17 in brain tissue. The method was carried out by centrifuging the brain tissue homogenate to obtain the 
supernatant. The following detection procedure was the same as above.

TTC Staining After anesthesia in rats, the brain tissue was quickly removed, and washed with physiological saline, and frozen at $-20^{\circ} \mathrm{C}$ for $15 \mathrm{~min}$, and placed on an ice bath table, and cut into one piece every $2 \mathrm{~mm}$, and cut into 5 pieces in succession. The brain slices were placed in 2\% TTC phosphate buffer wrapped in tin foil and incubated in an incubator at $37^{\circ} \mathrm{C}$ for about $20-30 \mathrm{~min}$. After staining, the brain slices were fixed in $4 \%$ paraformaldehyde solution for $24 \mathrm{~h}$, and the infarct volume was calculated by ImageJ software.

Western Blotting Analysis Total protein was extracted from intestinal tissue using T-PER tissue protein extraction reagent, and quantified by bicinchoninic acid (BCA); Different concentrations of separation gel and concentrated gel were arranged according to the molecular weight of the protein to be separated. Electrophoresis $(55 \mathrm{~V}$ voltage to protein MARK separation, separation gel with $110 \mathrm{~V}$ electrophoresis); Transfer: $200 \mathrm{~mA}$ constant current $120 \mathrm{~min}$ transfer protein to polyvinylidene difluoride (PVDF) membrane. Blocking: 5\% skim milk powder was sealed for $4 \mathrm{~h}$ at room temperature. Incubation primary antibody: Add 5\% skim milk powder to dilute the target protein ZO-1 $(1: 1000)$, Claudin-2 $(1: 1500)$ for $4^{\circ} \mathrm{C}$ overnight, remove the PVDF membrane, and wash with Phosphate Buffered Saline-Tween-20 (PBST) solution three times for $15 \mathrm{~min}$ each time. Add diluted horseradish peroxidase (HRP)-labeled $(1: 5000)$ secondary antibody, incubate for $1.5 \mathrm{~h}$ at room temperature, and wash with TBST solution 3 times for $15 \mathrm{~min}$ each time. Then add enhanced chemiluminescence (ECL) reagent. Imager 600 (General Electric, U.S.A.) exposure and development. $\beta$-Actin was used as an internal control.

Flow Cytometry Detect Treg Cells and $\gamma \delta$ T Cells First, the peripheral blood mononuclear cells should be separated and made into cell suspensions. The samples of counting up to $1 \times 10^{6} / \mathrm{mL}$, was dispensed into two tubes, and centrifuged, and discarded the supernatant, and washed twice with phosphate buffered saline (PBS). One hundred microliters of the liquid was taken, and the above mononuclear cells were mixed. The samples adding anti-CD4-fluorescein isothiocyanate (FITC) $2 \mu \mathrm{L}$, CD25-PE $5 \mu \mathrm{L}$ and CD3-FITC $2 \mu \mathrm{L}$, $\mathrm{TCR} \gamma \delta$-PE $5 \mu \mathrm{L}$, were incubated at $4^{\circ} \mathrm{C}$ for $30 \mathrm{~min}$ in the dark, and washed with PBS 2 times. After staining the surface of CD4 and CD25, the samples were added $1 \mathrm{~mL}$ of freshly prepared Fix/perm working solution, and incubated for $40-50 \mathrm{~min}$ in the dark at $4^{\circ} \mathrm{C}$. Then, the samples were washed in Perm/ Wash buffer, and added $5 \mu \mathrm{L}$ anti-Foxp3-APC to the cell suspension after rupture, and incubated for $30 \mathrm{~min}$ in the dark. After washing with Perm/Wash buffer, the stained cells were resuspended in $300 \mu \mathrm{L}$ of buffer and detected by flow cytometry (Flow Cytomics TM FC500, U.S.A.).

16S Ribosomal RNA (rRNA) Gene Sequencing Analysis After $7 \mathrm{~d}$ and $14 \mathrm{~d}$ of continuous administration, the fecal samples of the rats were collected by metabolic cage. After the experiment, the feces of the rats were placed in a sterile centrifuge tube and quickly stored in a refrigerator at $-80^{\circ} \mathrm{C}$. Microbial DNA was extracted from fecal samples using the E.Z.N.A. ${ }^{\circledR}$ soil DNA Kit (Omega Bio-tek, Norcross, GA, U.S.A.) according to manufacturer's protocols. The V3-V4 region of the 16S rRNA gene sequencing uses the upstream primer 338F (5'-ACTCTC ACGGGA GGC AGC AG-3') and downstream primer 806R (5'-GGACTACHVGGG TWTCT AA-3') was subjected to PCR amplification. Amplicons from each PCR sample were normalized to equimolar amounts and sequenced using $2 \times 300$ bp chemistry on an Illumina MiSeq platform (Illumina, San Diego, CA, U.S.A.) according to the standard protocols by Majorbio Bio-Pharm Technology Co., Ltd. (Shanghai, China). Raw reads were deposited into the NCBI Sequence Read Archive (SRA) database (Accession Number: SRP221538, http://www.ncbi.nlm.nih.gov/Traces/sra). Operational Taxonomic Units (OTU) is a unified mark that is artificially set for a certain classification unit (strain, genus, species, group, etc.) in order to facilitate analysis in phylogenetic or population genetics research. The RDP classifier Bayesian algorithm was used to classify the $97 \%$ similar level OTU representative sequence. At the genus level, the significance test between groups was performed. The single and double tail test was performed by two-tailed test. The significance level was set to 0.05 , and fdr was selected for multiple test correction. Beta diversity analysis was performed at the OTU level, and the Bray-curtis distance algorithm was used for Principal Component Analysis (PCA) to compare the differences in bacterial diversity of different groups. At the phylum level, we used the mean of the samples to detect the proportion of Bacteroidetes.

Immunohistochemistry Rat brain and small intestine and spleen tissue were fixed with 4\% paraformaldehyde for $12 \mathrm{~h}$. Paraffin sectioning was first performed, followed by dewaxing and hydration, then wash twice with PBS for 5 min each time. Antigen retrieval: the samples were sliceed into freshly prepared 3\% hydrogen peroxide solution, and blocked at room temperature for 5 to $10 \mathrm{~min}$, and washed 3 times with PBS for $5 \mathrm{~min}$ each time. Then add normal goat serum blocking solution, and leave the excess liquid at room temperature for 20 min. The I antibody was added dropwise at room temperature for $1 \mathrm{~h}$ or $4^{\circ} \mathrm{C}$ overnight, and washed three times with PBS for $2 \mathrm{~min}$ each time, and the biotinylated secondary antibody was added dropwise thereto at 20 to $37^{\circ} \mathrm{C}$ for $20 \mathrm{~min}$, and washed 3 times with $\mathrm{PBC}$ for $2 \mathrm{~min}$ each time. The reagent Strept Avidin-Biotin Complex (SABC) was added dropwise, 20 to $37^{\circ} \mathrm{C}$ for $20 \mathrm{~min}$, and washed 4 times with PBS for $5 \mathrm{~min}$ each time. 3,3'-Diaminobenzidine (DAB) color development: DAB color development kit or self-matching color developer (the degree of color development under the microscope) was used. The samples were distilled in water, and hematoxylin was counterstained for $2 \mathrm{~min}$, and hydrochloric acid alcohol was differentiated. Then the samples were dehydrated to transparency, sealed, and examined by microscope.

Frozen and paraffin sections are slightly different. Place the tissue block flat in a soft plastic cover or a special small box $(2 \mathrm{~cm}$ in diameter), then slowly place the special box into a small cup filled with liquid nitrogen, The tissue are quickly frozen into chunks in about 10-20s. Remove the tissue ice and immediately place it in the $-80^{\circ} \mathrm{C}$ refrigerator for later use. The frozen sections were air-dried at room temperature, fixed in cold acetone for $10 \mathrm{~min}$, and washed 3 times of PBS on a decolorizing shaker for $5 \mathrm{~min}$ each time. The remaining steps are same as the paraffin sections.

FMT Fresh feces from the Sham group, Sham+TQHXD group and Model+TQHXD group were collected into sterile tubes. Immediately after collecting the feces, they were dissolved in sterile water (to $200 \mathrm{mg} / \mathrm{mL}$ ), and centrifuged at $2000 \mathrm{rpm}$ for $10 \mathrm{~min}^{5)}$ Supernatant of each group was col- 
lected and given to the model rats separately by gavage of $2 \mathrm{~mL}$. The model group is given the same amount of sterile water every day. The FMT experimental process was carried out under SPF conditions.

Statistical Analysis All data were expressed as mean \pm standard deviation (S.D.). Each group was compared with the common control group by Dunnett $t$-test. The comparison among groups was analyzed by one-way ANOVA. The data between the two groups were compared by LSD $t$-test. The statistical analysis was performed with SPSS (SPSS, Inc, version23.0). $p<0.05$ was considered as statistically significant difference.

\section{RESULTS}

Effect of TQHXD on Neurological Scores after Stroke in Rats We performed neurological scores evaluation of rats in different groups at different time points. The behavioral scores of the model group were significantly higher than those of the sham operation group, indicating that cerebral ischemia caused certain damage to the neurological function of rats. After $7 \mathrm{~d}$ and $14 \mathrm{~d}$ of intragastric administration of TQHXD, the scores of behavioral scores at both time point were significantly lower in the TQHXD group (Fig. 1).

Effect of TQHXD on Stroke Induces Intestinal Microbiota Dysbiosis In order to prove the imbalance of intestinal microbes in rats with cerebral ischemia reperfusion and the improvement of TQHXD, we first performed a PCA of the intestinal flora in different groups of rats. Model groups 7 and $14 \mathrm{~d}$ are offset from the sham group, while TQHXD groups were different degrees of reversal after administration (Fig. 2a). Then we analyzed several major bacteria at the phylum level. We found that the proportion of Bacteroidetes in the model group $7 \mathrm{~d}$ was significantly higher than that in the sham operation group, and the TQHXD group was lower than the model group (Fig. 2b). Further, at the genus level, we performed a genus-specific analysis. Allobaculum and Bifidobacterium in the model group were significantly reduced compared with the sham group on 7 or $14 \mathrm{~d}$, and TQHXD was significantly increased after $14 \mathrm{~d}$ of administration. The model group Lactobacillus was significantly lower than the sham group at $7 \mathrm{~d}$, but the proportion of Lactobacillus even exceeded the sham group at $14 \mathrm{~d}$, which has the opposite trend compared with the results of model group 7d. This trend has improved after TQHXD administration, but there is no significant difference (Fig. 2c).

Effect of TQHXD on Stroke Induced Intestinal Epithelial Barrier We first observed the pathological section of the rat small intestine. Comparing of model groups of 7 and $14 \mathrm{~d}$ with sham group, the small intestine villi were destroyed, and the quantity was reduced, and the arrangement was sparse, and the goblet cells were significantly reduced. Among them, the intestinal pathological changes of the model group $7 \mathrm{~d}$ were the most obvious. TQHXD 7 and $14 \mathrm{~d}$ have different degrees of improvement compared with the model group (Fig. 3a). Then we used TEM to observe the tight junction ultrastructure of the intestinal barrier. We found that the tight junctions of the model group 7 and $14 \mathrm{~d}$ were dim or even disappeared, and the desmosome cells disappeared. The TQHXD group improved to different degrees at different time points (Fig. $3 b)$. At the protein level, we further verified that the $\mathrm{ZO}-1$ of model group was down-regulated at different time points, and

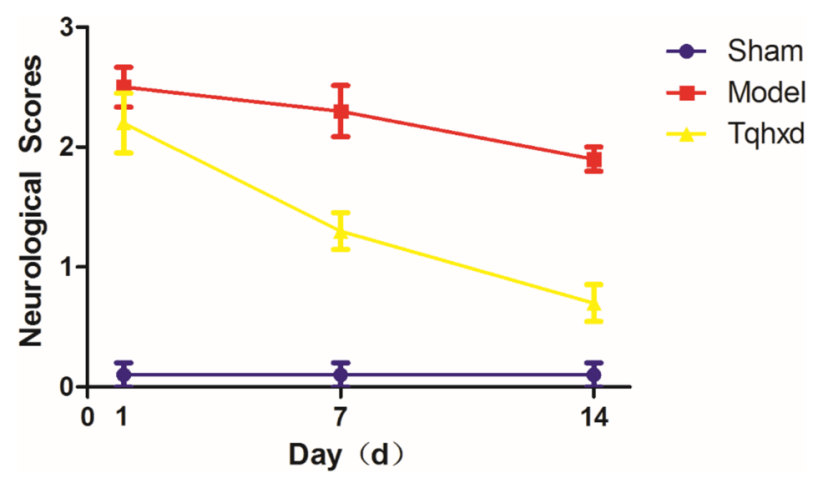

Fig. 1. The Effect of TQHXD on Neurological Deficit Scores at Different Time Points in Brain I/R Rat

Sham: Sham group; Model: Model group; tqhxd: TQHXD $(6 \mathrm{~g} / \mathrm{kg})$ group. Data are presented as means \pm S.D. $n=8$. (Color figure can be accessed in the online version.)

Claudin-2 expression was up-regulated, and TQHXD was reversed at different time points (Figs. 3c, d). We also measured the concentration of D-lactate, LPS and DAO in plasma. The results showed that the concentration of D-lactate, LPS and DAO increased in the model group at different time points. The TQHXD group has different degrees of reversal at different time points (Fig. 3e).

Effect of TQHXD on Pathological Changes of Brain Tissue after Stroke In brain tissue pathology, our results showed that the infarct volume was significantly increased in the Model group at 7 and $14 \mathrm{~d}$ compared with sham group, and the infarct volume was significantly reduced after TQHXD administration (Fig. 4a). The results of H\&E staining showed that compared with the sham operation group, the ischemic brain tissue of the model group was loose, the neurons were reduced and the center of the infarction was hyperemia and inflammatory cells were observed. Compared with the model group, the pathological changes of brain tissue in the TQHXD group were significantly alleviated (Fig. 4b).

Effect of TQHXD on the Changes of $\gamma \delta$ T Cells Caused by Intestinal Microecological Disorders after Stroke Immunohistochemical detection of intestinal and brain results showed that the proportion of $\gamma \delta \mathrm{T}$ cells in the model group was significantly higher than that in the Sham group, and there was a significant decrease after TQHXD treatment comparing with model group (Fig. 5a). In addition, we also detected changes in $\gamma \delta \mathrm{T}$ cells in peripheral blood and found the consistent results (Fig. 5b). In the subsequent detection of IL-17 in the ischemic hemisphere, IL-17 was significantly increased in the model group compared with the Sham group, and IL-17 expression was decreased in the TQHXD group comparing with model group (Fig. 5c).

Effect of TQHXD on the Changes of Treg Cells Caused by Intestinal Microecological Disorders after Stroke Immunohistochemical detection of intestine, brain and spleen showed that the Foxp3 expression was slightly increased in the model group compared with the Sham group. There was a significant increase of Foxp3 expression in TQHXD group comparing with model group (Fig. 6a). The expression of $\mathrm{CD} 4^{+} \mathrm{CD} 25^{+} \mathrm{Foxp}^{+}$in peripheral blood was the same as that in organ tissues (Fig. 6b). The expression of IL-10 in the cerebral ischemic hemisphere showed that IL-10 expression was significantly increased in the TQHXD group compared with 


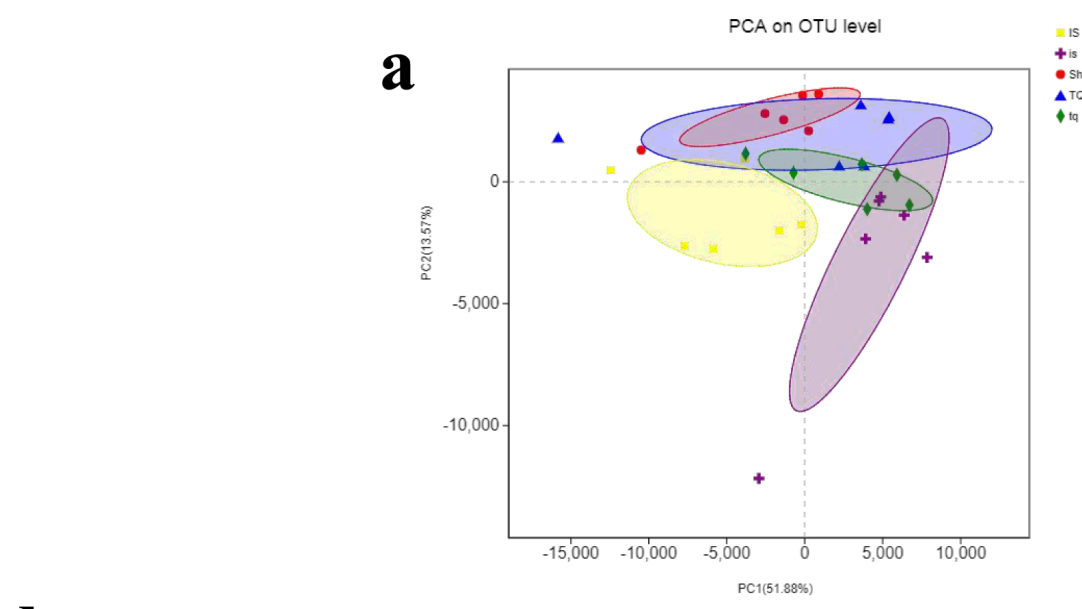

b
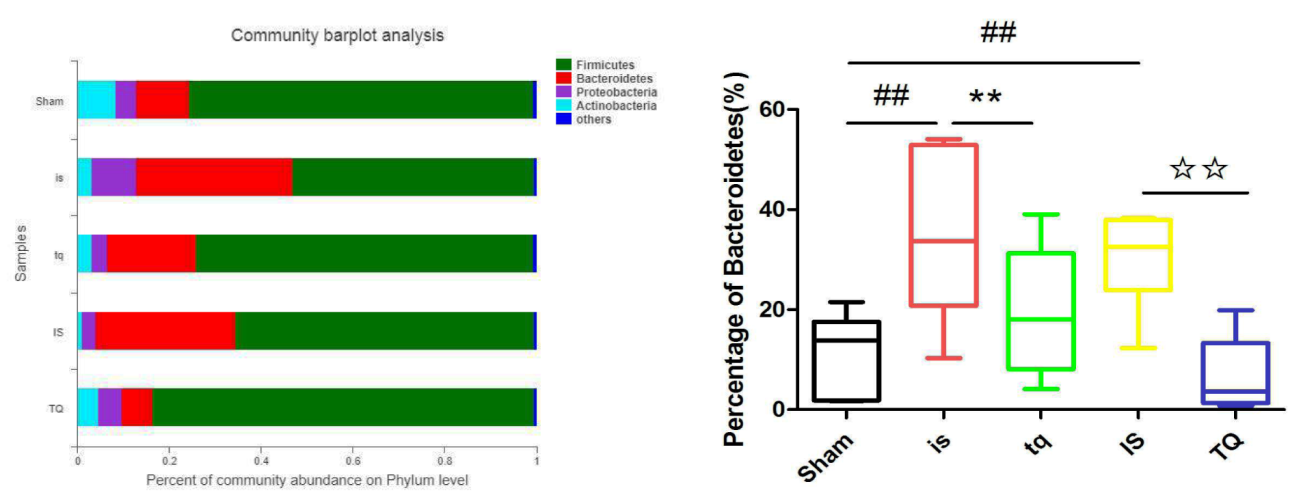

c

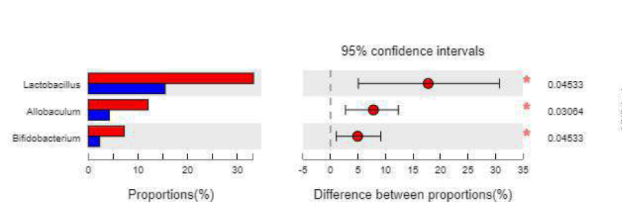

A

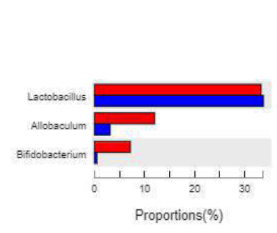

C
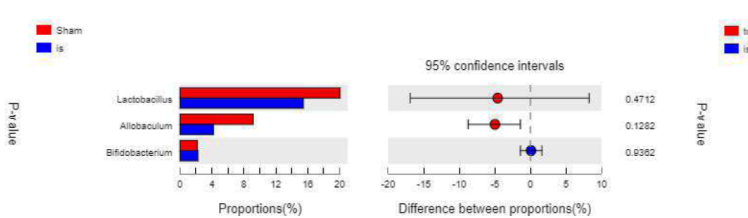

B
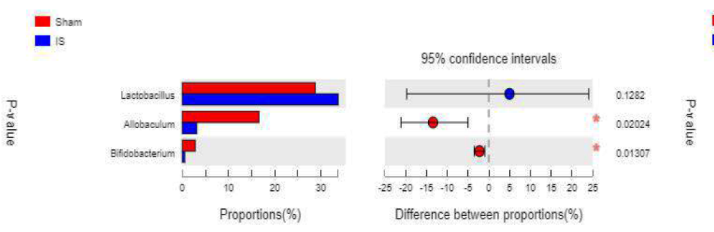

D

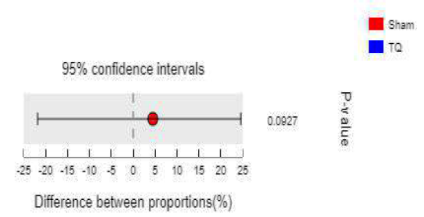

$\mathbf{E}$

Fig. 2. Effect of TQHXD on Stroke Induced Intestinal Flora Disorder

Sham: Sham group; is: Model group $7 \mathrm{~d}$; tq: TQHXD (6g/kg) group $7 \mathrm{~d}$; IS: Model group 14d; TQ: TQHXD (6g/kg) group $14 \mathrm{~d}$. A: Sham group vs. is group; B: tq group vs. is group; C: Sham group vs. IS group; D: TQ group vs. IS group; E: Sham group vs. TQ group. a, Intestinal flora PCA analysis. b, Difference analysis of the proportion of Bacteroidetes. c, Differential analysis of intestinal flora. \# $p<0.01, * * p<0.01, * p<0.05$, ${ }^{*} p<0.01$. Data are presented as means \pm S.D. $n=6$. (Color figure can be accessed in the online version.)

the Model group. But the increase of the model group relative to the Sham group was no significant difference (Fig. 6c).

Effect of Fecal Microbiota Transplantation on Poststroke Inflammatory Response Here, we tested the protec- tive effect of FMT as a possible therapeutic agent on poststroke rats. The results showed that the volume of cerebral infarction in the Sham (FMT) group and the Sham+TQHXD (FMT) group was significantly reduced compared with 


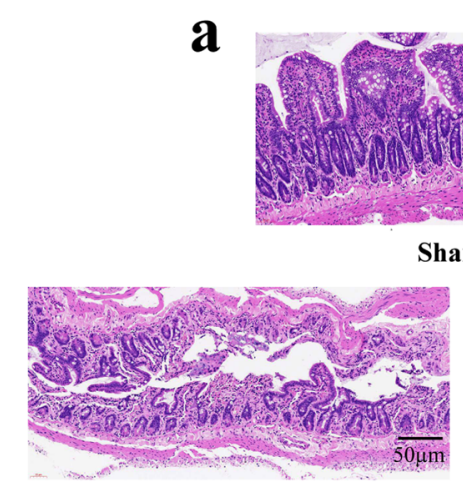

is

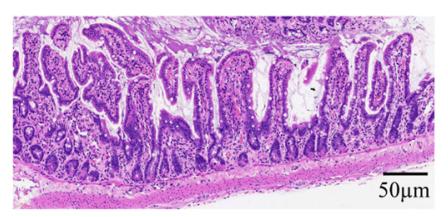

IS

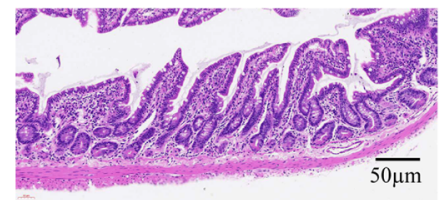

tq

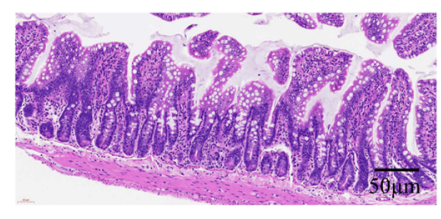

TQ

\section{b}

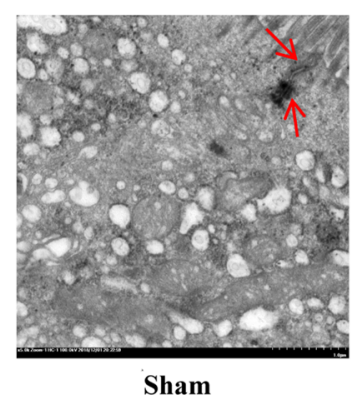

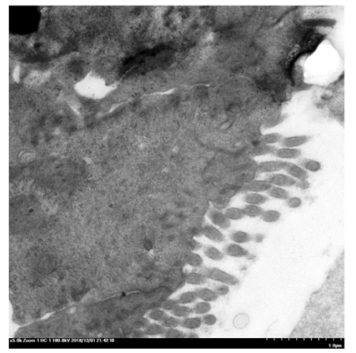

is

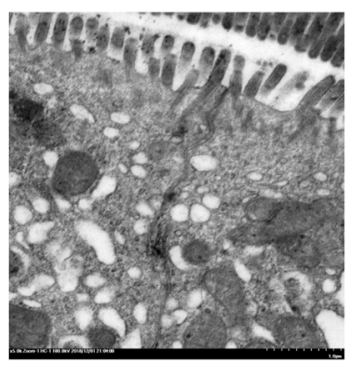

IS

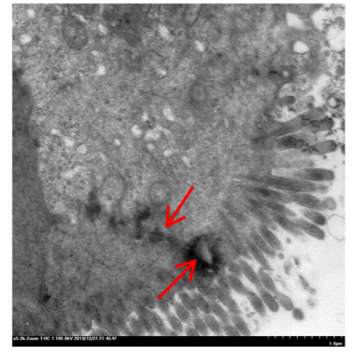

tq

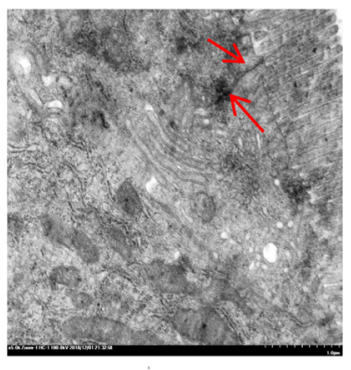

TQ c
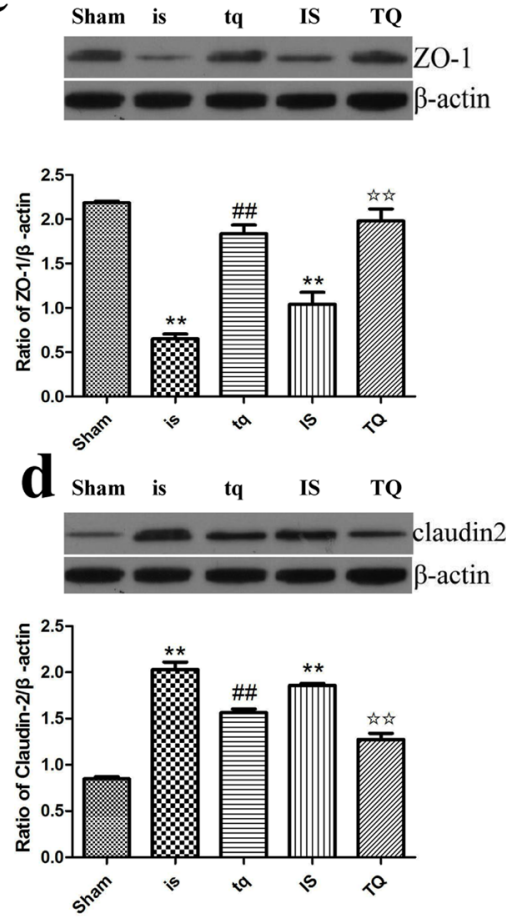

e
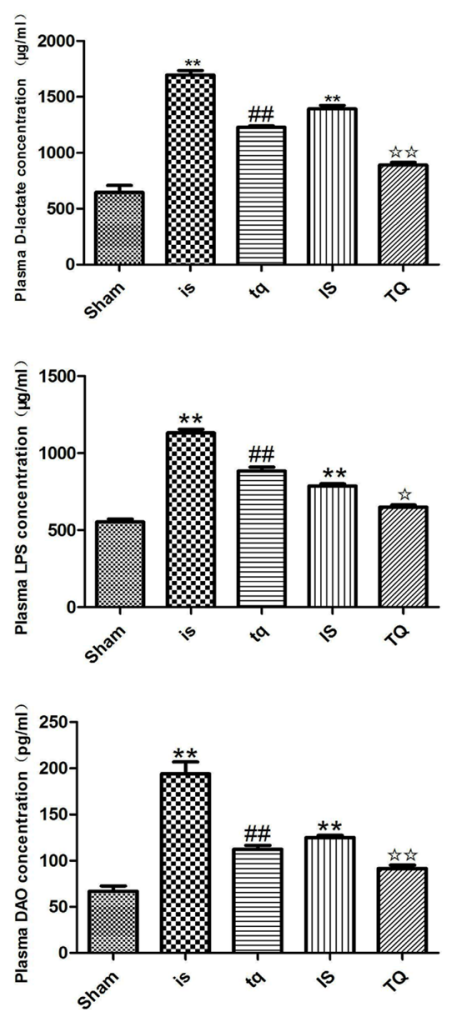

Fig. 3. Effect of TQHXD on Intestinal Permeability Changes after Stroke

Sham: Sham group; is: Model group $7 \mathrm{~d}$; tq: TQHXD (6g/kg) group 7d; IS: Model group 14d; TQ: TQHXD (6g/kg) group 14 d. a, Morphological observation of intestinal H\&E staining. Scale bar $=50 \mu \mathrm{m}(\times 200$ magnification). $\mathbf{b}$, TEM observation of intestinal tight junction structure. Arrow: tight junction structure. Scale bar $=1.0 \mu \mathrm{m}$ ( $\times 5000$ magnification) $\mathbf{c}$, d, WB detects the expression of tight junction protein ZO-1, Claudin-2 in the intestine. e, ELISA detection the concentration of D-lactate, LPS and DAO in plasma $(n=8)$. All bar graphics: ${ }^{\# \# ~} p<0.01$ versus is group, ${ }^{*} p<0.01$ versus sham group, $p<0.05$ versus IS group, ${ }^{2}$ 攻 $p<0.01$ versus IS group. Data are presented as means \pm S.D. (Color figure can be accessed in the online version.)

the model group (Fig. 7a). Although the Model+TQHXD (FMT) group can also reduce the volume of cerebral infarction, the results are not very significant. Compared with the model group, the amount of Treg cells in the intestine, brain and spleen tissues of the Sham (FMT) group and the Sham+TQHXD (FMT) group was significantly increased, 
a

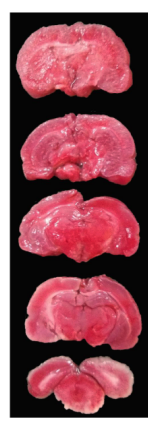

Sham

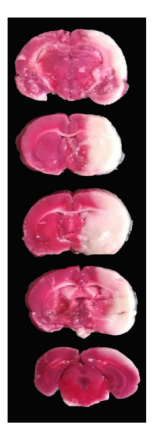

is

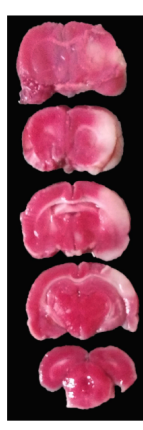

tq

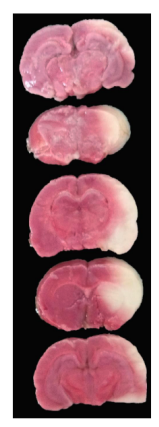

IS

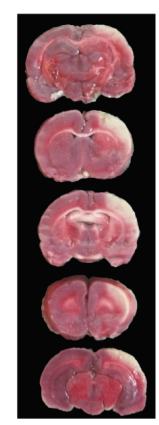

TQ

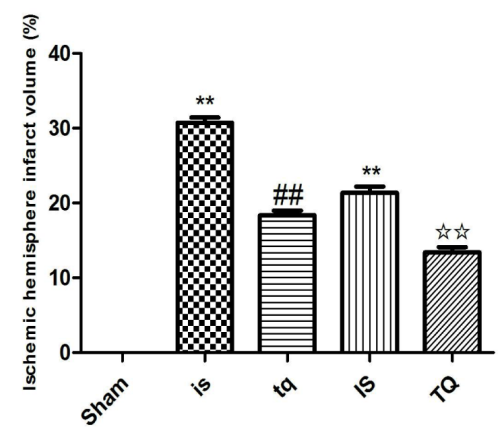

b

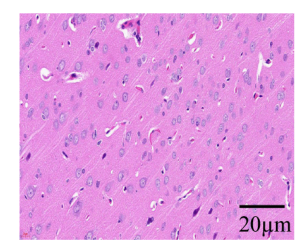

Sham

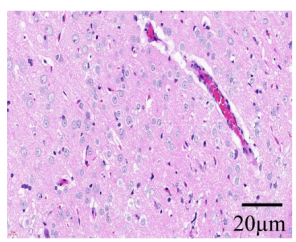

is

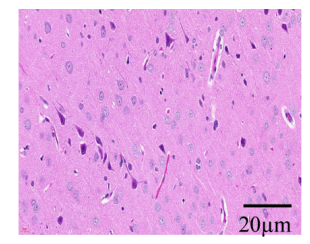

tq

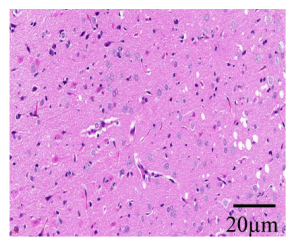

IS

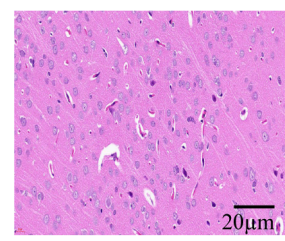

TQ

Fig. 4. Effect of TQHXD on Ischemic Hemisphere

Sham: Sham group; is: Model group $7 \mathrm{~d}$; tq: TQHXD $(6 \mathrm{~g} / \mathrm{kg})$ group $7 \mathrm{~d}$; IS: Model group $14 \mathrm{~d}$; TQ: TQHXD $(6 \mathrm{~g} / \mathrm{kg})$ group $14 \mathrm{~d}$. a, TTC staining for detecting cerebral infarction volume. ${ }^{\#} p<0.01$ versus is group, $* * p<0.01$ versus sham group, ${ }^{*} p<0.01$ versus IS group. b, Observation of pathological changes of brain tissue by H\&E staining. Scale bar $=20 \mu \mathrm{m}(\times 400$ magnification). (Color figure can be accessed in the online version.)

while the amount of $\gamma \delta$ T cells in the intestine and brain tissues reduced significantly. There were no significant differences between the Sham (FMT) group and the Sham+TQHXD (FMT) group (Figs. 7b, c). Next, we examined the inflammatory factors secreted by the Treg cells and $\gamma \delta \mathrm{T}$ cells. The Sham (FMT) group and the Sham+TQHXD (FMT) group showed a significant increase of IL-10 production in the brain tissue compared with the Model group, and IL-17 production was significantly reduced. There were no significant differences between the Sham (FMT) group and the Sham+TQHXD (FMT) group (Figs. 7d, e).

\section{DISCUSSION}

The construct method of animal model used in this experiment has the advantages of no craniotomy, affirmative effect and small damage. It is currently recognized as an ideal method for establishing a focal cerebral ischemia model. ${ }^{30,31)}$ Additionally, we used the Longa five-level four-point behavioral score to evaluate and screen successful model rats for experimentation. The results of this experiment show that compared with the sham group, the neurological deficit score of model group is significantly increased. Thus the score results indicate that the ischemia-reperfusion model has been successfully established and caused certain damage. Moreover, compared with the model group, the neurological deficit score was significantly decreased after TQHXD administration and the behavior was significantly restored and the ischemic injury was alleviated as well (Fig. 1).

In general, maintaining normal intestinal physiological function plays an important role in the regulation of immunity and the antagonism of pathogenic microbial invasion. ${ }^{23,32}$ ) More and more studies have shown that intestinal flora is closely related to the occurrence and development of central nervous system diseases. ${ }^{25,33-37)}$ Actually, after the stroke, the body's immune function declines and causes intestinal flora disorder. On the contrary, the imbalanced intestinal flora also affects the stroke-related immune response, severity, stroke outcome and related complications. ${ }^{38)}$ Relevant literature studies show that sympathetic nerve activity after middle cerebral artery occlusion can cause reduced intestinal motility and changes in flora composition. ${ }^{39)}$ Therefore, adjusting the intestinal function after stroke to approach normalization plays a positive role in the outcome of stroke.

The presented results show that at the OTU level, the model group has significant changes in principal components compared with sham group according to the PCA diagram (Fig. 2a). The sample points of the TQHXD group are closer to the distance of the sham group sample points than the model group. This suggested that TQHXD can regulate the disordered intestinal flora to approach normal level. At the phylum level, the ratios of the Bacteroidetes in the model group $7 \mathrm{~d}$ and $14 \mathrm{~d}$ are increased in different degrees compared with the sham group, which is consistent with the study of Singh et al. ${ }^{5)}$ The increase in the proportion of Bacteroidetes may be an influencing factor affecting the outcome of stroke. TQHXD 
a
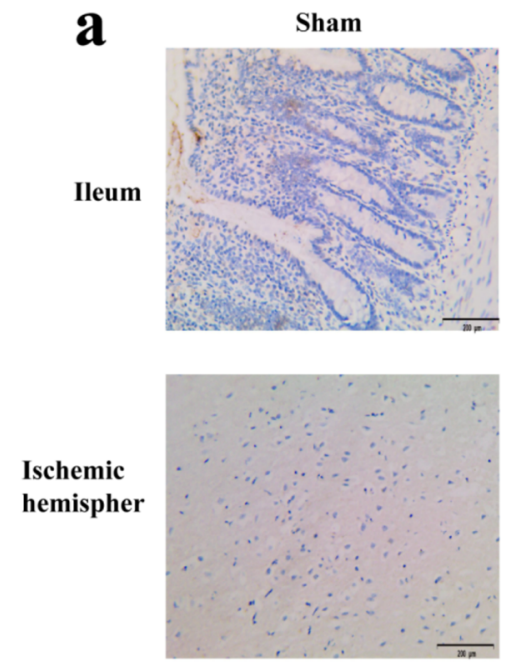

Model
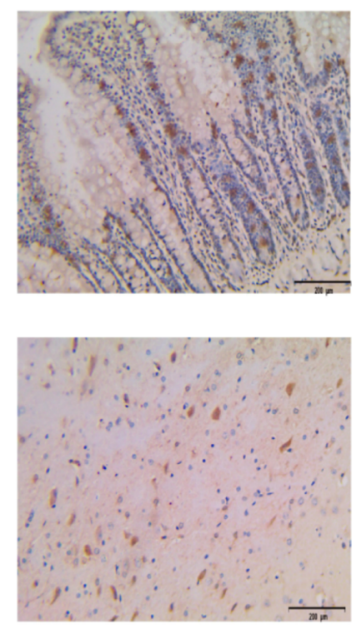

TQHXD
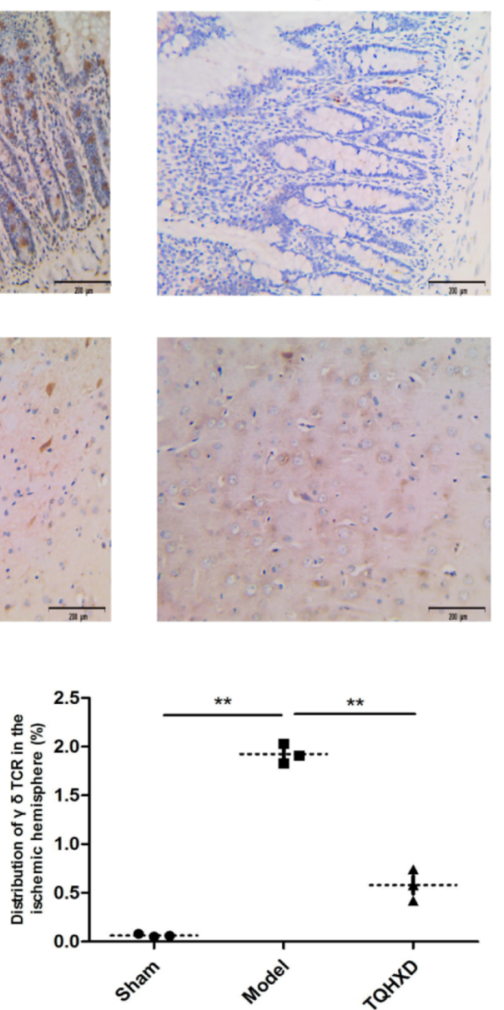

b

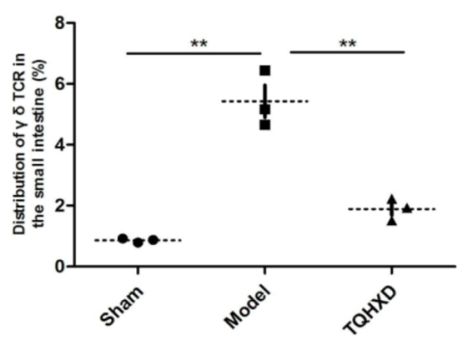

peripheral blood
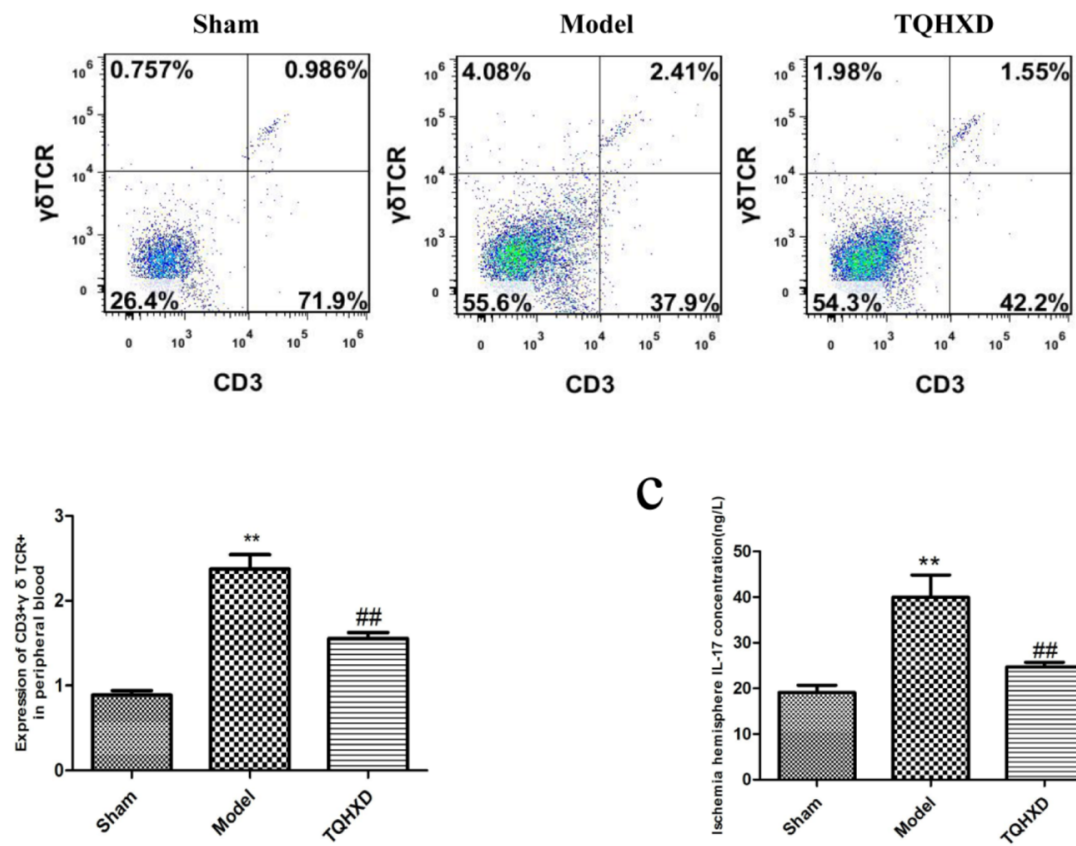

C

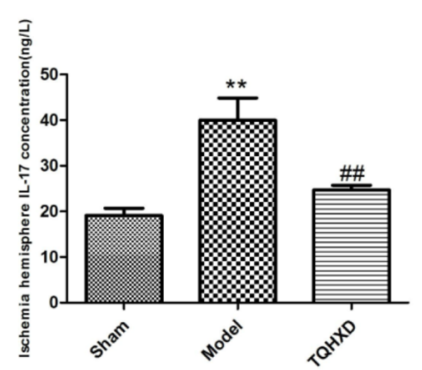

Fig. 5. Effect of TQHXD on the Expression of $\gamma \delta \mathrm{T}$ Cells after Stroke

a, Immunohistochemical detection of $\gamma \delta \mathrm{TCR}$ expression in brain and intestine. Scale bar $=100 \mu \mathrm{m}\left(\times 200\right.$ magnification). b. Detection of $\mathrm{CD}^{+} \gamma \delta \mathrm{TCR}{ }^{+}$expression in peripheral blood by flow cytometry. c, ELISA detects the expression of IL-17 in the ischemic hemisphere $(n=8)$. All graphics: $* * p<0.01$ versus sham group, \# $p<0.01$ versus Model group. Data are presented as means \pm S.D. (Color figure can be accessed in the online version.)

can reverse the increase in the proportion of intestinal Bacteroidetes after stroke (Fig. 2b). In addition these findings on poststroke dysbiosis are consistent with recent reports describing changes in intestinal microbiota composition in stroke patients. ${ }^{40,41)}$ We also found that the abundances of Bifidobacterium and Allobaculum in the model group 7 and $14 \mathrm{~d}$ were different with the sham group in the genus-specific analysis. TQHXD can regulate the reduction of Bifidobacterium and $\mathrm{Al}$ lobaculum after stroke (Fig. 2c).

Normal intestinal function in addition to the balance of the intestinal flora, the intestinal epithelial barrier function is also crucial. If the intestinal barrier function is impaired, intestinal 

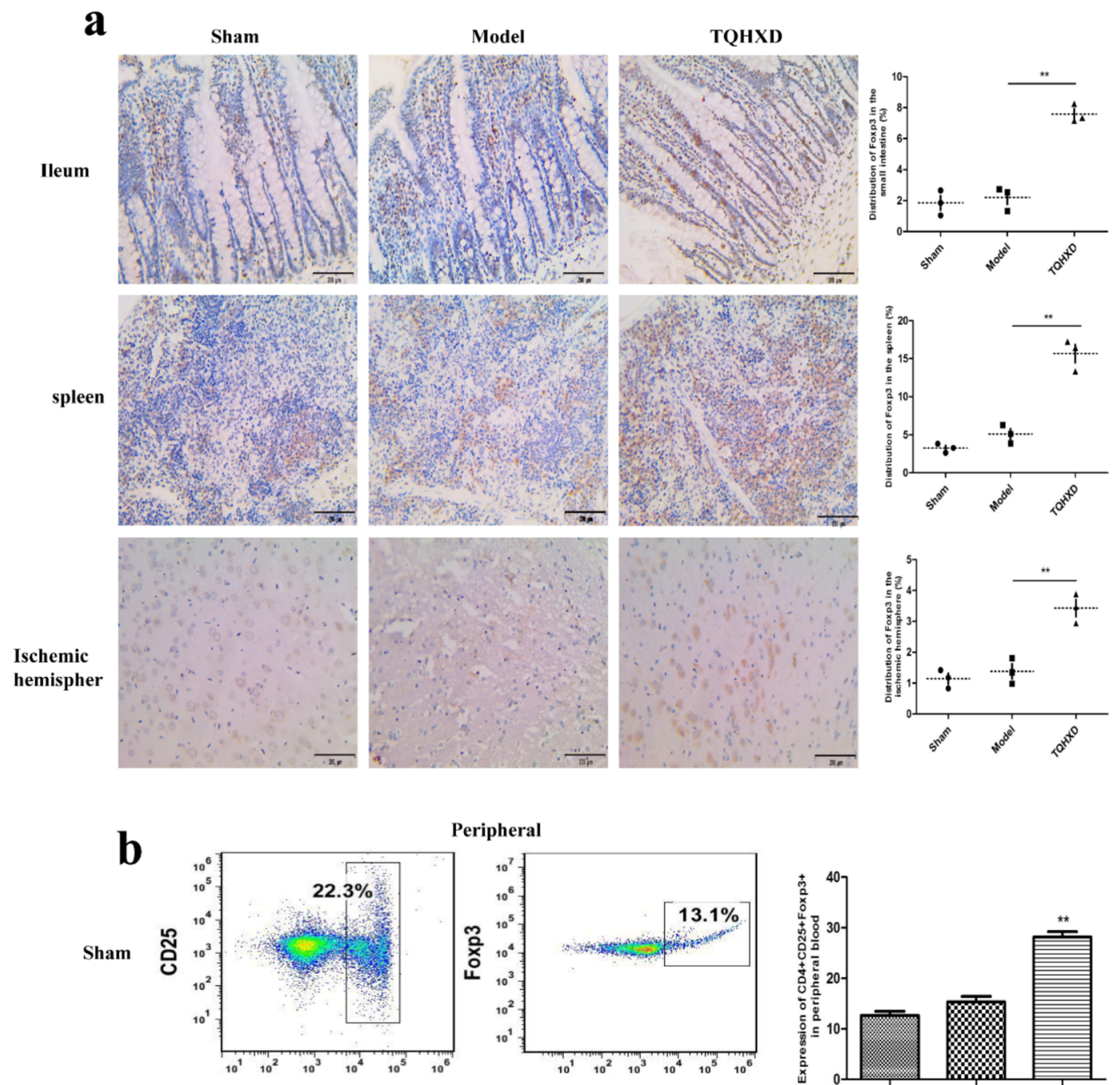

Peripheral
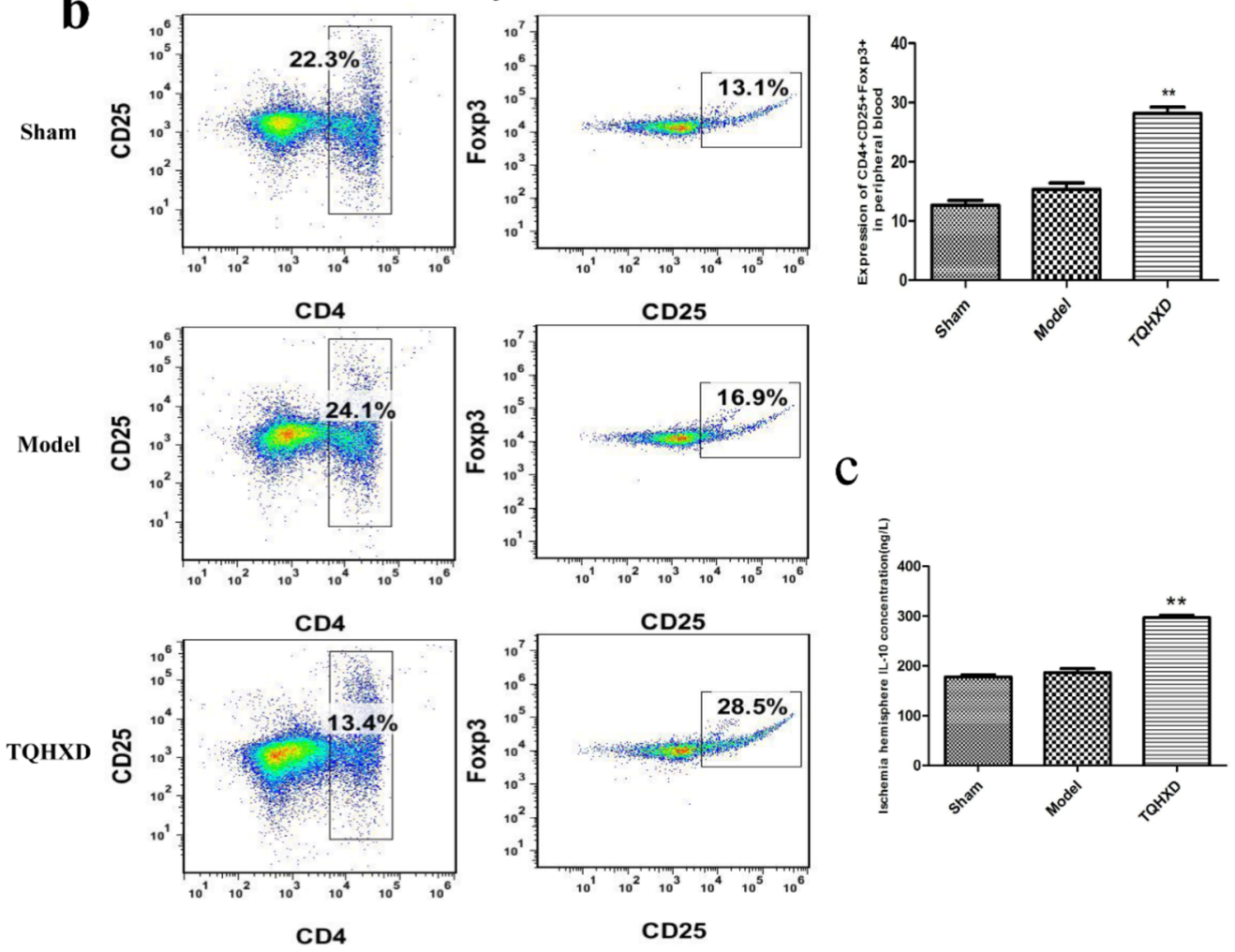

Fig. 6. Effect of TQHXD on the Expression of Treg Cells after Stroke (Choose 7d Time Point for Testing)

a, Immunohistochemical detection of Foxp $3^{+}$expression in brain, intestine and spleen. Scale bar $=100 \mu \mathrm{m}(\times 200$ magnification $) . \mathbf{b}$, Detection of CD $4^{+} \mathrm{CD} 25^{+}$Foxp $3^{+}$ex pression in peripheral blood by flow cytometry. $\mathbf{c}$, ELISA detects the expression of IL-10 in the ischemic hemisphere $(n=8)$. All graphics: $* * p<0.01$ versus Model group. Data are presented as means \pm S.D. (Color figure can be accessed in the online version.)

bacteria and LPS can lead to systemic inflammatory response syndrome and even life-threatening. Therefore, prevention and treatment of intestinal barrier failure has become an important topic in the current medical field, ${ }^{42,43)}$ studies have shown that ischemic stroke can cause an increase in intestinal barrier permeability. ${ }^{44-46)}$

Whether in morphological observation or protein level detection, the intestinal barrier of the Model group was de- 


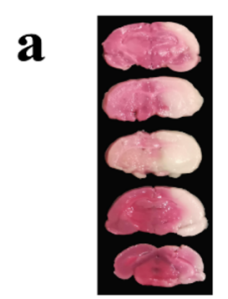

b
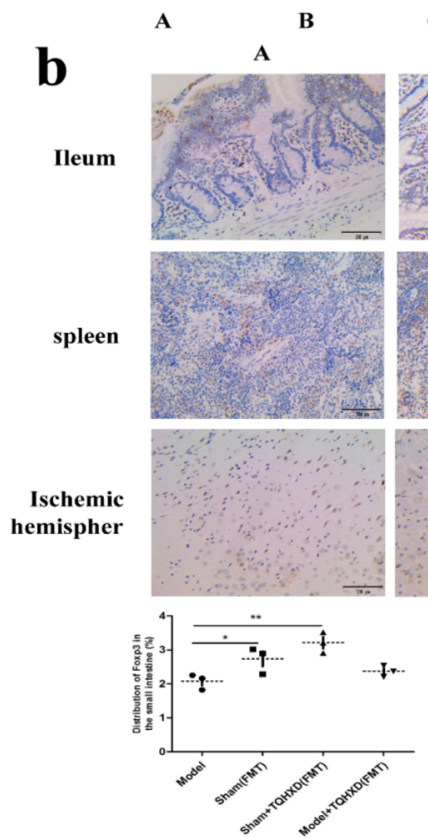

c

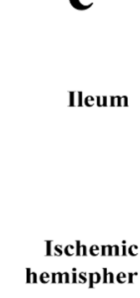

A
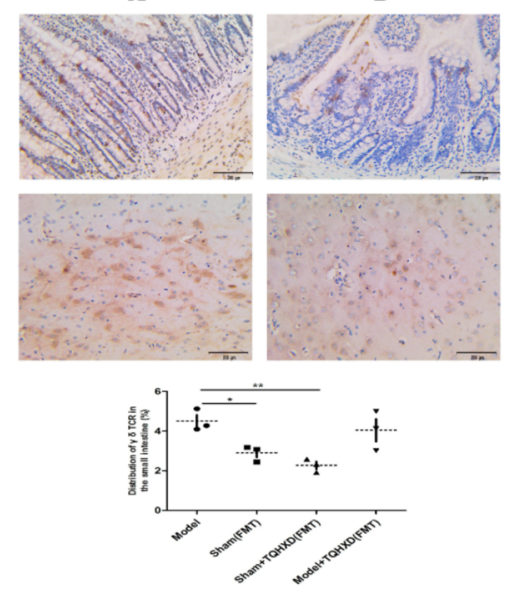

d
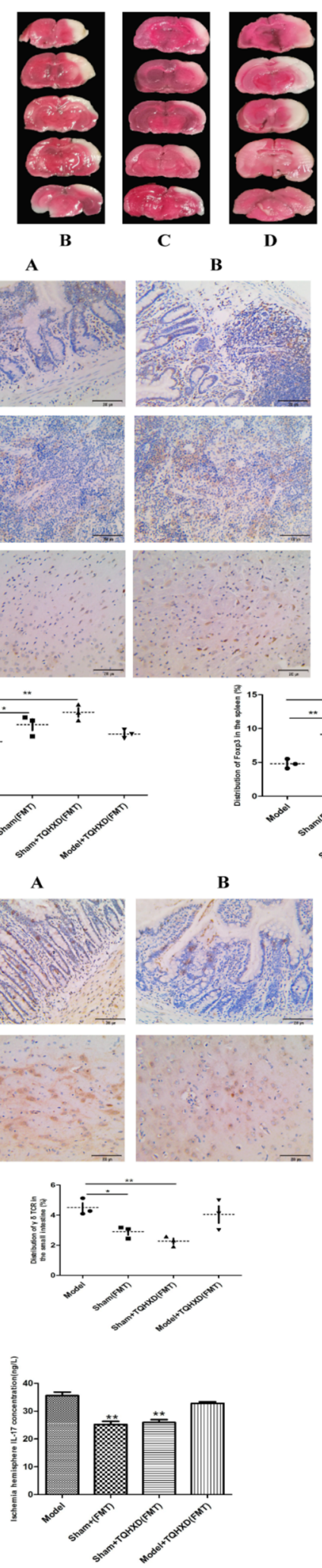

D

B
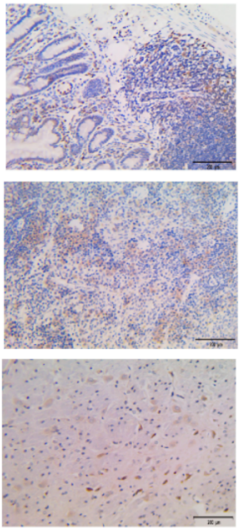

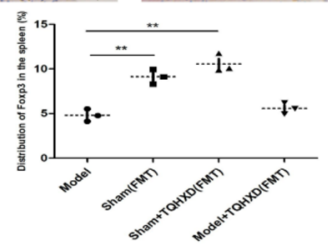

C
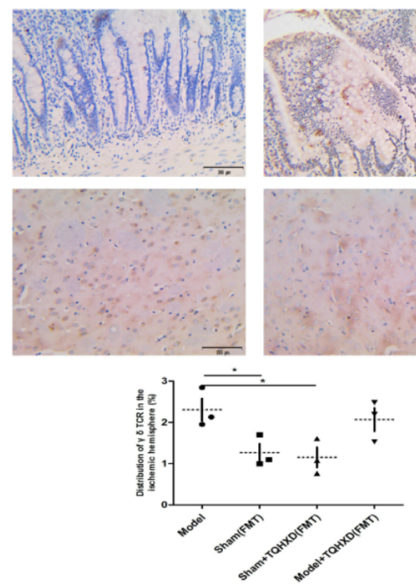

e

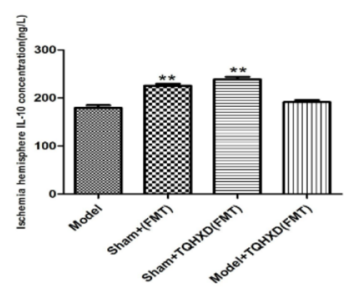

Fig. 7. Effect of Fecal Microbial Transplantation on Stroke

A: Model group, B: Sham (FMT), C: Sham+TQHXD (FMT), D: Model+TQHXD (FMT). a, TTC staining for detecting cerebral infarction volume. b, Immunohistochemical detection of Foxp $3^{+}$expression in brain, intestine and spleen. Scale bar $=100 \mu \mathrm{m}(\times 200$ magnification $)$. c, Immunohistochemical detection of $\gamma \delta$ TCR expression in brain and intestine. Scale bar $=100 \mu \mathrm{m}(\times 200$ magnification). d, e, ELISA detects the expression of IL-10 and IL-17 in the ischemic hemisphere $(n=8)$. All graphics: $*_{p}<0.05, * * p<0.01$ versus Model group. Data are presented as means \pm S.D. (Color figure can be accessed in the online version.)

stroyed and the permeability was enhanced. As the intestinal epithelial barrier was destroyed, intestinal bacteria and their metabolites are transferred to the blood circulation, resulting in an increase in the levels of D-lactic acid, LPS, and DAO in plasma. ${ }^{47-49)}$ According to ELISA, we also found that the concentration of D-lactate, LPS and DAO in plasma was significantly increased in the model group compared with the sham group, and all these effects were reversed after TQHXD 
treatment (Fig. 3).

Khailova et al..$^{50)}$ found that Bifidobacterium can improve intestinal integrity in a rat model of colitis. Jurgen Karczewski's ${ }^{51)}$ study showed that Lactobacillus regulated tight junction proteins and had a protective effect on the epithelial barrier. According to their research and our experimental results, the reduction of Bifidobacterium and the disorder of Lactobacillus in the intestinal tract after stroke may be the cause of the destruction of the intestinal barrier. Bifidobacterium is increased after TQHXD treatment, and the destruction of the intestinal barrier has also been regulated. Toll-like receptor 4 (TLR4) is an important binding ligand for LPS, ${ }^{52,53)}$ activated TLR4 induces the production of a variety of pro-inflammatory cytokines, causing and maintaining low levels of inflammation. ${ }^{53)}$ LPS also disrupted the blood-brain barrier and is associated with LPS down regulation of the tight junction proteins Occludin, Zonula occludens-1, and their mRNA expression. When the blood-brain barrier is damaged, its permeability increases, and harmful substances in the blood easily enter brain tissue, which aggravates brain cell damage. ${ }^{54)}$ In the previous study, ${ }^{27)}$ the research team had confirmed the protective effect of TQHXD on blood-brain barrier disruption after stroke. In this experiment, we detected a significant increase in LPS in the blood of the model group, while TQHXD can reduce the expression of LPS, which is also a progress to previous studies. According to the previous pharmacodynamics related indicators, we found that the disease symptoms of $7 d$ after stroke was more obvious than the $14 \mathrm{~d}$ and it has a certain effect after $7 \mathrm{~d}$ of administration of TQHXD. Therefore, we chose the experimental study of the inflammatory response at this time point of $7 \mathrm{~d}$. A previous study has reported that a combination of Puerariae Lobatae radix and Chuan Xiong rhizoma effectively treats cerebral ischemic stroke through remodeling of the intestinal microbiota. ${ }^{55)} \mathrm{TQHXD}$ contains other drugs in addition to Chuan Xiong, and our results show that TQHXD can be used in addition to regulate the disturbance of intestinal flora and the destruction of the intestinal epithelial barrier, and also have a protective effect on the intestinal microecological disturbance-induced inflammatory response. We found that the $\gamma \delta \mathrm{T}$ cells of intestinal tissue increased significantly after stroke in rats (Fig. 5a). $\gamma \delta \mathrm{T}$ cells are located in the intestinal epithelium and the major $\mathrm{T}$ cell subsets involved in innate immune function. ${ }^{56)} \gamma \delta \mathrm{T}$ cells secrete IL-17, which acts as a pro-inflammatory factor, causing tissue inflammatory cell infiltration by contacting the body to specific immunity system and the innate immune system, leading to tissue destruction. Benakis et al. ${ }^{25)}$ found that IL-17+ $\gamma \delta \mathrm{T}$ cells in the intestine of ischemic stroke migrated to the meninges and deposited, and were associated with the degree of ischemic stroke. Intestinal flora imbalance can promote $\gamma \delta \mathrm{T}$ cell activation, ${ }^{57)}$ which in turn aggravates the inflammatory response in patients with ischemic stroke. Studies have shown ${ }^{58)}$ that IL-17 plays a key role in post-stroke injury. Knockout of IL-17 gene in mice can significantly reduce infarct volume of stroke. We also found a significant increase in $\gamma \delta \mathrm{T}$ cells in peripheral blood and brain tissue of the Model group (Figs. $5 \mathrm{a}, \mathrm{b})$. In normal brain tissue, $\gamma \delta \mathrm{T}$ cells are hardly found, but a large amount of $\gamma \delta \mathrm{T}$ cells are detected in ischemic brain tissue of rats after stroke, which indicated that a large number of $\gamma \delta \mathrm{T}$ cells are secreted in the intestine after stroke, then through the blood circulation to reach the ischemic hemisphere of the brain tissue and secreted IL-17 (Fig. 5c), which led to further deterioration of the stroke. After TQHXD intervention, we found that Treg cells in the intestinal tissue of rats increased significantly, while the spleen, peripheral blood and ischemic hemisphere Treg cells increased too (Figs. 6a, b). Subsequently, we detected a significant increase in the IL-10 of the ischemic hemisphere (Fig. 6c), and the related literature ${ }^{25}$ ) showed that IL-10 can inhibit IL-17 secreted by $\gamma \delta$ T cells. In our experiments $\gamma \delta \mathrm{T}$ cells and IL-17 were significantly lower than those in the Model group after TQHXD administration, indicating that TQHXD can inhibit the inflammatory response by increasing the expression of Treg cells, which affects the outcome of the stroke. In this study, we found that the Treg cells and IL-10 in the Model group are not significantly different with the Sham group, while the increase in $\gamma \delta$ T cells and IL-17 in the Model group was significant. Therefore, a slight increase in Treg cells and IL-10 in the Model group may be the result of self-regulation of the body.

In addition, studies have shown ${ }^{59,60)}$ that Bifidobacterium and Lactobacillus can increase the expression of Foxp3 and Treg cells. In our study, TQHXD was able to improve the reduction of Bifidobacterium in the model group. The immune response after stroke was more complicated. It had a complicated relationship with the disorder of intestinal flora, the destruction of intestinal barrier, and the peripheral immune organs. Further research is needed for the specific mechanism research.

To further demonstrate the effect of the gut microbiota on stroke outcomes, we used the FMT approach. Finally, we found that Sham (FMT) group, Sham+TQHXD (FMT) group after the fecal microbiota transplantation compared with the Model group, Foxp3 in the ischemic side hemisphere, spleen and intestinal tissue increased significantly (Fig. 7b). IL-10 of ischemic side hemisphere are also been a significant increase (Fig. 7e). Singh et al. ${ }^{5)}$ found that the amount of Foxp3 in the ischemic hemisphere or spleen was significantly increased in the stroke mice after transplantation of normal mouse feces, and the volume of cerebral infarction was reduced, this is consistent with our findings (Fig. 7a). In addition, the Model+TQHXD (FMT) group received FMT treatment showed that although Foxp3 and IL-10 increased, $\gamma \delta$ T cells and IL-17 decreased, but there was no significant compared with the Model group. Although the intestinal micro-ecology of the model rats after TQHXD treatment was adjusted, it was not enough to have a substantial effect on the intestinal microbes of the Model rats, or it would take longer. Fecal microbial transplantation experiments and related studies have shown that intestinal microbes do affect the outcome of stroke. ${ }^{25)}$

It has been reported that Treg/Th17 ratio changes are also indicators of post-stroke inflammatory response. ${ }^{61,62)}$ In addition to $\mathrm{T}$ cell polarization, monocytes, microglia and macrophages are also involved in microbial-mediated effects on stroke outcomes. ${ }^{5,63)}$ Intestinal microbial disorders and immune inflammatory reactions after stroke are a relatively complex process, and the links between specific indicators and the specific functional mechanisms of the flora need further study. Next the research team can then use the relevant gene knockout mice and sterile mice to explore the relationship between the specific microflora and disease after stroke, and the specific mechanism of peripheral immune organs involved in post-stroke inflammatory response. 
In conclusion, our results reveal that TQHXD inhibits the inflammatory response by regulating the disorder of the intestinal flora and the destruction of the intestinal barrier after stroke, thus affecting the outcome of stroke. Our results would provide new ideas for clinical treatment of stroke and prevention of intestinal dysfunction after stroke.

Acknowledgments The study was supported by the National Natural Science Foundation of China (No. 81773933), Academic assistance program for the top-notch innovative talents from universities in 2017 provided by Anhui Province Office of Education (gxbjZD15), the Key Project of Natural Science Research in Universities of Anhui Province in 2019 (No. KJ2019A0434).

Conflict of Interest The authors declare no conflict of interest.

\section{REFERENCES}

1) Sun $\mathrm{K}, \mathrm{Hu} \mathrm{Q}$, Zhou $\mathrm{CM}$, Xu XS, Wang F, Hu BH, Zhao XY, Chang $\mathrm{X}$, Chen CH, Huang P, An LH, Liu YY, Fan JY, Wang CS, Yang L, Han JY. Cerebralcare Granule, a Chinese herb compound preparation, improves cerebral microcirculatory disorder and hippocampal CA1 neuron injury in gerbils after ischemia-reperfusion. J. Ethnopharmacol., 130, 398-406 (2010).

2) Liu X, Wang Z, Wang P, Yu B, Liu Y, Xue Y. Green tea polyphenols alleviate early BBB damage during experimental focal cerebral ischemia through regulating tight junctions and PKCalpha signaling. BMC Complement. Altern. Med., 13, 187 (2013).

3) Bennett DA, Krishnamurthi RV, Barker-Collo S, Forouzanfar MH, Naghavi M, Connor M, Lawes CM, Moran AE, Anderson LM, Roth GA, Mensah GA, Ezzati M, Murray CJ, Feigin VL. The global burden of ischemic stroke: findings of the GBD 2010 study. Glob. Heart, 9, 107-112 (2014)

4) Kim HA, Whittle SC, Lee S, Chu HX, Zhang SR, Wei Z, Arumugam TV, Vinh A, Drummond GR, Sobey CG. Brain immune cell composition and functional outcome after cerebral ischemia: comparison of two mouse strains. Front. Cell. Neurosci., 8, 365 (2014).

5) Singh V, Roth S, Llovera G, Sadler R, Garzetti D, Stecher B, Dichgans M, Liesz A. Microbiota dysbiosis controls the neuroinflammatory response after stroke. J. Neurosci., 36, 7428-7440 (2016).

6) Oyama N, Winek K, Backer-Koduah P, Zhang T, Dames C, Werich M, Kershaw O, Meisel C, Meisel A, Dirnagl U. Exploratory investigation of intestinal function and bacterial translocation after focal cerebral ischemia in the mouse. Front. Neurol., 9, 937 (2018).

7) Nagpal R, Yadav H. Bacterial translocation from the gut to the distant organs: an overview. Ann. Nutr. Metab., 71 (Suppl. 1), 11-16 (2017).

8) Li N, Wang X, Sun C, Wu X, Lu M, Si Y, Ye X, Wang T, Yu X, Zhao X, Wei N, Wang X. Change of intestinal microbiota in cerebral ischemic stroke patients. BMC Microbiol., 19, 191 (2019).

9) Schwartz M, Raposo C. Protective autoimmunity: a unifying model for the immune network involved in CNS repair. Neuroscientist, 20, $343-358$ (2014)

10) Lopes Pinheiro MA, Kooij G, Mizee MR, Kamermans A, Enzmann G, Lyck R, Schwaninger M, Engelhardt B, de Vries HE. Immune cell trafficking across the barriers of the central nervous system in multiple sclerosis and stroke. Biochim. Biophys. Acta, 1862, 461-471 (2016).

11) Liesz A, Karcher S, Veltkamp R. Spectratype analysis of clonal $T$ cell expansion in murine experimental stroke. J. Neuroimmunol., 257, 46-52 (2013).

12) Xiong XY, Liu L, Yang QW. Functions and mechanisms of microglia/macrophages in neuroinflammation and neurogenesis after stroke. Prog. Neurobiol., 142, 23-44 (2016).

13) Li P, Wang L, Zhou Y, Gan Y, Zhu W, Xia Y, Jiang X, Watkins S, Vazquez A, Thomson AW, Chen J, Yu W, Hu X. C-C Chemokine receptor type 5 (CCR5)-mediated docking of transferred tregs protects against early blood-brain barrier disruption after stroke. $J$. Am. Heart Assoc., 6, e006387 (2017).

14) Shu L, Xu CQ, Yan ZY, Yan Y, Jiang SZ, Wang YR. Post-stroke microglia induce sirtuin2 expression to suppress the anti-inflammatory function of infiltrating regulatory $\mathrm{T}$ Cells. Inflammation, $\mathbf{4 2}$, 1968-1979 (2019).

15) Liesz A, Dalpke A, Mracsko E, Antoine DJ, Roth S, Zhou W, Yang $\mathrm{H}$, Na SY, Akhisaroglu M, Fleming T, Eigenbrod T, Nawroth PP, Tracey KJ, Veltkamp R. DAMP signaling is a key pathway inducing immune modulation after brain injury. J. Neurosci., 35, 583-598 (2015).

16) Zhang BJ, Men XJ, Lu ZQ, Li HY, Qiu W, Hu XQ. Splenectomy protects experimental rats from cerebral damage after stroke due to anti-inflammatory effects. Chin. Med. J., 126, 2354-2360 (2013).

17) Gelderblom M, Melzer N, Schattling B, Gob E, Hicking G, Arunachalam P, Bittner S, Ufer F, Herrmann AM, Bernreuther C, Glatzel M, Gerloff C, Kleinschnitz C, Meuth SG, Friese MA, Magnus T. Transient receptor potential melastatin subfamily member 2 cation channel regulates detrimental immune cell invasion in ischemic stroke. Stroke, 45, 3395-3402 (2014).

18) Arpaia N, Campbell C, Fan X, Dikiy S, van der Veeken J, deRoos P, Liu H, Cross JR, Pfeffer K, Coffer PJ, Rudensky AY. Metabolites produced by commensal bacteria promote peripheral regulatory $\mathrm{T}$ cell generation. Nature, 504, 451-455 (2013).

19) Atarashi K, Tanoue $T$, Oshima $K$, et al. Treg induction by a rationally selected mixture of Clostridia strains from the human microbiota. Nature, 500, 232-236 (2013).

20) Omenetti S, Pizarro TT. The Treg/Th17 Axis: A dynamic balance regulated by the gut microbiome. Front. Immunol., 6, 639 (2015).

21) Gomez-Eguilaz M, Ramon-Trapero JL, Perez-Martinez L, Blanco JR. The microbiota-gut-brain axis and its great projections. Rev. Neurol., 68, 111-117 (2019).

22) Collins SM, Surette M, Bercik P. The interplay between the intestinal microbiota and the brain. Nat. Rev. Microbiol., 10, 735-742 (2012).

23) Winek K, Meisel A, Dirnagl U. Gut microbiota impact on stroke outcome: Fad or fact? J. Cereb. Blood Flow Metab., 36, 891-898 (2016).

24) Bondarenko VM, Riabichenko EV. Intestinal-brain axis. Neuronal and immune-inflammatory mechanisms of brain and intestine pathology. Zh. Mikrobiol. Epidemiol. Immunobiol., 2013, 112-120 (2013).

25) Benakis C, Brea D, Caballero S, Faraco G, Moore J, Murphy M, Sita G, Racchumi G, Ling L, Pamer EG, Iadecola C, Anrather J. Commensal microbiota affects ischemic stroke outcome by regulating intestinal gammadelta T cells. Nat. Med., 22, 516-523 (2016).

26) Xu FQ, Wang N, Liu JQ, Zhou A, Duan JA, Ding AW, Zhu Q. Studies on fingerprints of Tongqiao Huoxue Decoction by HPLC. Chin. J. Exp. Trad. Med. Form, 14, 1-4 (2008).

27) Li L, Wang N, Jin Q, Wu Q, Liu Y, Wang Y. Protection of TongQiao-Huo-Xue Decoction against cerebral ischemic injury through reduction blood-brain barrier permeability. Chem. Pharm. Bull., 65, 1004-1010 (2017)

28) Wang N, Deng Y, Wei W, Song L, Wang Y. Serum containing Tongqiaohuoxue decoction suppresses glutamate-induced PC12 cell injury. Neural Regen. Res., 7, 1125-1131 (2012).

29) Longa EZ, Weinstein PR, Carlson S, Cummins R. Reversible middle cerebral artery occlusion without craniectomy in rats. Stroke, 20, 84-91 (1989).

30) McBride DW, Zhang JH. Precision stroke animal models: the permanent MCAO model should be the primary model, not transient MCAO. Transl. Stroke Res., 2017, 397-404 (2017).

31) Ao LY, Li WT, Zhou L, Yan YY, Ye AQ, Liang BW, Shen WY, Zhu 
X, Li YM. Therapeutic effects of JLX-001 on ischemic stroke by inducing autophagy via AMPK-ULK1 signaling pathway in rats'. Brain Res. Bull., 153, 162-170 (2019).

32) Backhed F, Ley RE, Sonnenburg JL, Peterson DA, Gordon JI. Host-bacterial mutualism in the human intestine. Science, 307, 1915-1920 (2005).

33) Cryan JF, Dinan TG. Mind-altering microorganisms: the impact of the gut microbiota on brain and behaviour. Nat. Rev. Neurosci., 13, 701-712 (2012).

34) Tascilar N, Irkorucu O, Tascilar O, Comert F, Eroglu O, Bahadir B, Cakmak GK, Ankarali H, Sayan H. Bacterial translocation in experimental stroke: what happens to the gut barrier? Bratisl. Lek Listy, 111, 194-199 (2010).

35) Borre YE, O'Keeffe GW, Clarke G, Stanton C, Dinan TG, Cryan JF. Microbiota and neurodevelopmental windows: implications for brain disorders. Trends Mol. Med., 20, 509-518 (2014).

36) Ochoa-Reparaz J, Mielcarz DW, Begum-Haque S, Kasper LH. Gut, bugs, and brain: role of commensal bacteria in the control of central nervous system disease. Ann. Neurol., 69, 240-247 (2011).

37) Winek K, Dirnagl U, Meisel A. The gut microbiome as therapeutic target in central nervous system diseases: implications for Stroke. Neurotherapeutics, 13, 762-774 (2016).

38) $\mathrm{Xu} \mathrm{R}$, Tan $\mathrm{C}$, Zhu J, Zeng $\mathrm{X}$, Gao $\mathrm{X}$, Wu Q, Chen Q, Wang $\mathrm{H}$, Zhou H, He Y, Pan S, Yin J. Dysbiosis of the intestinal microbiota in neurocritically ill patients and the risk for death. Crit. Care, 23, 195 (2019).

39) Houlden A, Goldrick M, Brough D, Vizi ES, Lenart N, Martinecz $\mathrm{B}$, Roberts IS, Denes A. Brain injury induces specific changes in the caecal microbiota of mice via altered autonomic activity and mucoprotein production. Brain Behav. Immun., 57, 10-20 (2016).

40) Yin J, Liao SX, He Y, Wang S, Xia GH, Liu FT, Zhu JJ, You C Chen Q, Zhou L, Pan SY, Zhou HW. Dysbiosis of gut microbiota with reduced trimethylamine- $n$-oxide level in patients with largeartery atherosclerotic stroke or transient ischemic attack. J. Am. Heart Assoc., 4, e002699 (2015).

41) Karlsson FH, Fak F, Nookaew I, Tremaroli V, Fagerberg B, Petranovic D, Backhed F, Nielsen J. Symptomatic atherosclerosis is associated with an altered gut metagenome. Nat. Commun., 3, 1245 (2012).

42) Lachar J, Bajaj JS. Changes in the microbiome in cirrhosis and relationship to complications: hepatic encephalopathy, spontaneous bacterial peritonitis, and sepsis. Semin. Liver Dis., 36, 327-330 (2016).

43) Wang N, Han Q, Wang G, Ma WP, Wang J, Wu WX, Guo Y, Liu L, Jiang XY, Xie XL, Jiang HQ. Resveratrol protects oxidative stressinduced intestinal epithelial barrier dysfunction by upregulating heme oxygenase-1 expression. Dig. Dis. Sci., 61, 2522-2534 (2016).

44) Stanley D, Mason LJ, Mackin KE, Srikhanta YN, Lyras D, Prakash MD, Nurgali K, Venegas A, Hill MD, Moore RJ, Wong CH. Translocation and dissemination of commensal bacteria in post-stroke infection. Nat. Med., 22, 1277-1284 (2016).

45) Wen SW, Wong CHY. An unexplored brain-gut microbiota axis in stroke. Gut Microbes, 8, 601-606 (2017).

46) Wen SW, Shim R, Ho L, Wanrooy BJ, Srikhanta YN, Prame Kumar K, Nicholls AJ, Shen SJ, Sepehrizadeh T, de Veer M, Srikanth VK, Ma H, Phan TG, Lyras D, Wong CHY. Advanced age promotes colonic dysfunction and gut-derived lung infection after stroke. Aging Cell, 18, e12980 (2019).

47) Fukudome I, Kobayashi M, Dabanaka K, Maeda H, Okamoto K, Okabayashi T, Baba R, Kumagai N, Oba K, Fujita M, Hanazaki K. Diamine oxidase as a marker of intestinal mucosal injury and the effect of soluble dietary fiber on gastrointestinal tract toxicity after intravenous 5-fluorouracil treatment in rats. Med. Mol. Morphol., 47, 100-107 (2014).

48) Shi H, Wu B, Wan J, Liu W, Su B. The role of serum intestinal fatty acid binding protein levels and D-lactate levels in the diagnosis of acute intestinal ischemia. Clin. Res. Hepatol. Gastroenterol., 39, 373-378 (2015).
49) Stevens BR, Goel R, Seungbum K, Richards EM, Holbert RC, Pepine CJ, Raizada MK. Increased human intestinal barrier permeability plasma biomarkers zonulin and FABP2 correlated with plasma LPS and altered gut microbiome in anxiety or depression. Gut, 67, 1555-1557 (2018).

50) Khailova L, Dvorak K, Arganbright KM, Halpern MD, Kinouchi T, Yajima M, Dvorak B. Bifidobacterium bifidum improves intestinal integrity in a rat model of necrotizing enterocolitis. Am. J. Physiol. Gastrointest. Liver Physiol., 297, G940-G949 (2009).

51) Karczewski J, Troost FJ, Konings I, Dekker J, Kleerebezem M, Brummer RJ, Wells JM. Regulation of human epithelial tight junction proteins by Lactobacillus plantarum in vivo and protective effects on the epithelial barrier. Am. J. Physiol. Gastrointest. Liver Physiol., 298, G851-G859 (2010).

52) Patra S, Muthuraman MS, Meenu M, Priya P, Pemaiah B. Antiinflammatory effects of royal poinciana through inhibition of tolllike receptor 4 signaling pathway. Int. Immunopharmacol., 34, 199-211 (2016).

53) Nighot M, Al-Sadi R, Guo S, Rawat M, Nighot P, Watterson MD, Ma TY. Lipopolysaccharide-Induced increase in intestinal epithelial tight permeability is mediated by toll-like receptor 4/Myeloid differentiation primary response 88 (MyD88) activation of myosin light chain kinase expression. Am. J. Pathol., 187, 2698-2710 (2017).

54) McCusker RH, Kelley KW. Immune-neural connections: how the immune system's response to infectious agents influences behavior. J. Exp. Biol., 216, 84-98 (2013).

55) Chen R, Wu P, Cai Z, Fang Y, Zhou H, Lasanajak Y, Tang L, Ye L, Hou C, Zhao J. Puerariae lobatae radix with chuanxiong rhizoma for treatment of cerebral ischemic stroke by remodeling gut microbiota to regulate the brain-gut barriers. J. Nutr. Biochem., 65, 101-114 (2019).

56) Prinz I, Silva-Santos B, Pennington DJ. Functional development of gammadelta T cells. Eur. J. Immunol., 43, 1988-1994 (2013).

57) Zhang L, Huang Y, Lin Y, Shan Y, Tan S, Cai W, Li H, Zhang B, Men X, Lu Z. Anti-inflammatory effect of cholera toxin B subunit in experimental stroke. J. Neuroinflammation, 13, 147 (2016).

58) Shichita T, Sugiyama Y, Ooboshi H, Sugimori H, Nakagawa R, Takada I, Iwaki T, Okada Y, Iida M, Cua DJ, Iwakura Y, Yoshimura A. Pivotal role of cerebral interleukin-17-producing gammadeltaT cells in the delayed phase of ischemic brain injury. Nat. Med., 15, 946-950 (2009).

59) Konieczna P, Groeger D, Ziegler M, Frei R, Ferstl R, Shanahan F, Quigley EM, Kiely B, Akdis CA, O’Mahony L. Bifidobacterium infantis 35624 administration induces Foxp3 $\mathrm{T}$ regulatory cells in human peripheral blood: potential role for myeloid and plasmacytoid dendritic cells. Gut, 61, 354-366 (2012).

60) Saez-Lara MJ, Gomez-Llorente C, Plaza-Diaz J, Gil A. The role of probiotic lactic acid bacteria and bifidobacteria in the prevention and treatment of inflammatory bowel disease and other related diseases: a systematic review of randomized human clinical trials. BioMed Res. Int., 2015, 505878 (2015)

61) Liesz A, Suri-Payer E, Veltkamp C, Doerr H, Sommer C, Rivest S, Giese T, Veltkamp R. Regulatory T cells are key cerebroprotective immunomodulators in acute experimental stroke. Nat. Med., 15, 192-199 (2009)

62) Kleinschnitz C, Schwab N, Kraft P, Hagedorn I, Dreykluft A, Schwarz T, Austinat M, Nieswandt B, Wiendl H, Stoll G. Early detrimental T-cell effects in experimental cerebral ischemia are neither related to adaptive immunity nor thrombus formation. Blood, $\mathbf{1 1 5}$, 3835-3842 (2010).

63) Erny D, Hrabe de Angelis AL, Jaitin D, Wieghofer P, Staszewski O, David E, Keren-Shaul H, Mahlakoiv T, Jakobshagen K, Buch T, Schwierzeck V, Utermohlen O, Chun E, Garrett WS, McCoy KD, Diefenbach A, Staeheli P, Stecher B, Amit I, Prinz M. Host microbiota constantly control maturation and function of microglia in the CNS. Nat. Neurosci., 18, 965-977 (2015). 